\title{
The impact of a repeated bout of eccentric exercise on muscular strength, muscle soreness and creatine kinase
}

\author{
L. L. Smith PhD, M. G. Fulmer MA, D. Holbert* PHD, M. R. McCammon MA, \\ J. A. Houmard PhD, D. D. Frazer ${ }^{\dagger}$ MD, E. Nsien ${ }^{\ddagger}$ MD and R. G. Israel EdD
}

Human Performance Laboratory, *Department of Biostatistics, ${ }^{\dagger}$ Department of Rheumatology and ${ }^{\ddagger}$ Department of Internal Medicine, East Carolina University, Greenville, USA

\begin{abstract}
The purpose of this study was to determine if there were any beneficial or detrimental effects regarding delayed onset muscle soreness (DOMS), serum creatine kinase (CK), and maximum concentric strength at $80 \%$ of $1-R M_{\text {conc, }}$ if a bout of eccentric exercise was repeated at $48 \mathrm{~h}$ after an initial bout. A secondary purpose was to determine whether unaccustomed eccentrics might affect plasma cholesterol (TC). Twenty-six men were randomly assigned to a control (Group 1) or experimental group (Group 2). Both groups performed three sets (12 repetitions per set) of the eccentric phase of a chest press, at $80 \%$ of one repetition maximum (1-RMconc); Group 2 repeated this exercise $48 \mathrm{~h}$ later. DOMS and CK were measured before, and every $24 \mathrm{~h}$ for 8 days after; TC was measured before, and every $24 \mathrm{~h}$ for 4 days. Maximum strength during the concentric phase of a chest press $\left(1-\mathrm{RM}_{\text {conc }}\right)$ was measured before and at 48-h intervals after. $A$ repeated measures analysis of variance revealed a significant time effect $(P<0.05)$ for DOMS, CK and strength, but no significant difference between groups $(P<0.05)$. An interesting finding was the significant $(P<0.05)$ reduction in TC at 24, 48 and $72 \mathrm{~h}$, after exercise in both groups, which we hypothesized was associated with cellular repair. From these results we concluded that when a bout of eccentrics is repeated $48 \mathrm{~h}$ after an initial bout, there is no change in the characteristic time-course and/or intensity of DOMS, CK or 1-RM conc.
\end{abstract}

Keywords: Eccentric muscle action, delayed onset muscle soreness, strength, total cholesterol

Delayed onset muscle soreness (DOMS) is a sensation of discomfort or pain that occurs in response to unaccustomed exercise, or in response to large increases in the volume of exercise. It is first felt between $8-24 \mathrm{~h}$ after exercise, peaks in intensity between 24 and $72 \mathrm{~h}$ and usually disappears by 5 days ${ }^{1-4}$. DOMS is associated with connective ${ }^{5-8}$ and contractile $^{3,4}$ tissue microtrauma, resulting from high tensions generated during the eccentric phase of a movement ${ }^{1}$. Complete healing does occur, although little is known about this aspect ${ }^{2}$.

Address for correspondence: Dr Lucille L. Smith PhD, Human Performance Laboratory, 371 Sports Medicine Building, Greenville, North Carolina 27858, USA

(C) 1994 Butterworth-Heinemann Ltd 0306-3674/94/040267-05
The general recommendation concerning exercise during the period of muscle soreness ${ }^{2}$ is to 'ignore the sensations and work through the pain'. In addition, anecdotal reports suggest that exercise during this period might hasten dissipation of the soreness. However, there is no scientific evidence to substantiate or refute these claims.

Friden and colleagues ${ }^{9}$ reported that in the days following eccentrically biased exercise, traumatized muscle fibres are swollen and presumably weaker and more vulnerable to injury. Leadbetter ${ }^{10}$ suggested that after any sports injury, there is a susceptible period during which there is a greater risk of reinjury. Therefore, exercise during this early stage might be detrimental to recovery either because of reinjury or interference with healing ${ }^{10,11}$. Therefore, the main objective of this study was to determine whether repeating a bout of eccentrics at $48 \mathrm{~h}$ would exacerbate, alleviate or have no effect on DOMS, creatine kinase (CK) and strength.

Increases in serum levels of CK are used as an indirect marker of the microtrauma associated with $\mathrm{DOMS}^{3,4}$. However, there appear to be no markers related to the process of healing. Since cholesterol is a component of cell membrane ${ }^{12}$, and since blood cholesterol levels are temporarily reduced in response to trauma ${ }^{13}$, and after surgery ${ }^{14}$, we were interested in whether there would be a reduction in total cholesterol levels in response to microtrauma induced by eccentric activity. Therefore a second objective of this study was to examine changes in plasma cholesterol levels in response to one and two bouts of eccentric exercise.

\section{Subjects and methods}

Twenty-six healthy, untrained men volunteered for this study. None had performed any weight training for at least 3 months before the study. Subjects were screened using a medical history form and were required to complete an informed consent. Subjects were then randomly assigned to Group 1 (performed one bout of eccentric exercise, $n=13$ ) or Group 2 (performed two bouts of eccentric exercise, $n=13$ ). For means and standard errors of physical characteristics for groups 1 and 2, see Table 1. A standard $t$ test 
Table 1. Subject demographics

\begin{tabular}{|c|c|c|}
\hline & $\begin{array}{l}\text { Group } 1 \\
(n=13)\end{array}$ & $\begin{array}{l}\text { Group } 2 \\
(n=13)\end{array}$ \\
\hline $\begin{array}{l}\text { Age (years) } \\
\text { Height (cm) } \\
\text { Weight (kg) } \\
\text { Body fat (\%) } \\
\text { (five-site skinfold) } \\
1 \text { Repetition max (kg) } \\
\text { (concentric) }\end{array}$ & $\begin{array}{r}20.6(0.6) \\
178.8(1.5) \\
77.8(3.6) \\
14.6(1.3)\end{array}$ & $\begin{array}{r}21.6(0.6) \\
179.3(2.3) \\
79.1(4.2) \\
14.7(1.8)\end{array}$ \\
\hline
\end{tabular}

Values are mean(s.e.)

was used to test for group differences. No significant differences $(P>0.05)$ were found between the groups for any of the demographic variables.

\section{Strength assessments}

The Cybex Eagle Chest Press Machine (Cybex, Division of Lumex, Ronkonkoma, New York, USA) was used for all strength assessments. One concentric repetition maximum (1-RM $\left.\mathrm{Ronc}_{\text {c }}\right)$ was determined during a preliminary visit. Subjects warmed up by performing ten repetitions of the concentric phase of a chest press at a standard resistance of $20 \mathrm{~kg}$. A 2-min rest followed the warm-up. The subject then estimated the maximum amount of weight that he could push (concentric action) through a full range of motion. The 1-RM $\mathrm{Ronc}_{\text {c }}$ was determined in three to five trials for all subjects; this was considered the baseline for subsequent strength measures.

$1-\mathrm{RM}_{\text {conc }}$ was assessed at 48,96 and $192 \mathrm{~h}$ after exercise. At each time the subject was required to perform a warm-up at a load equal to $30 \%$ of their $1-\mathrm{RM}_{\text {conc }}$ After a 2-min rest the resistance was adjusted to the level recorded on the previous visit; if necessary, the resistance was increased or decreased.

\section{Eccentric exercise protocol}

Exercise was performed on the Cybex Eagle Chest Press Machine. The eccentric exercise protocol involved performance of the eccentric, lowering phase of the chest press with two safety spotters performing the positive, lifting phase. On the day of the eccentric exercise, subjects warmed up as described in the previous section. Subjects then performed three sets of 12 repetitions of the eccentric phase of a chest press at an intensity equal to $80 \%$ of their previously determined $1-\mathrm{RM}_{\text {conci }}$ there was a 2 -min rest period between each set. This number of repetitions and intensity were selected since they represent what is commonly prescribed when an individual initiates a weight-training programme ${ }^{15}$. Group 1 performed one bout of exercise; Group 2 repeated the exercise $48 \mathrm{~h}$ after the initial bout. For Group 2, assessments of plasma creatine kinase, strength and muscle soreness were made before subjects performed the second bout of exercise. Subjects were informed of which group they were in, after the 48-h assessments.

\section{Delayed onset muscle soreness (DOMS) ratings}

DOMS measurements were made before, and every $24 \mathrm{~h}$ following, the bout of eccentric exercise, for 8 days. Subjects were shown a soreness scale with a range of $1-10\left(1=\right.$ no soreness, $10=$ very sore $\left.^{16,17}\right)$. They were instructed to palpate muscles of the chest and upper arm and assign a number between 1 and 10 that best represented their overall rating of soreness.

\section{Creatine kinase (CK)}

CK was assessed before the initial bout of exercise, and then every $24 \mathrm{~h}$ for 8 days. On each occasion, upon arriving in the laboratory, subjects sat quietly for $5 \mathrm{~min}$. Blood was then drawn from an anticubital vein into nontreated vacutainers. The blood $(3 \mathrm{ml})$ was allowed to clot at room temperature for $10 \mathrm{~min}$ and centrifuged for $15 \mathrm{~min}$. Serum was separated and frozen at $-20^{\circ} \mathrm{C}$ for subsequent analysis. Total CK was determined spectrophotometrically, in duplicate, at $25^{\circ} \mathrm{C}$, using a commercially available kit (Sigma Diagnostics, St. Louis, Missouri, USA).

\section{Total cholesterol (TC)}

TC was assessed for 13 subjects in Group 1 and 13 subjects in Group 2. A 10-ml venous blood sample was drawn into Vacutainer serum separator tubes (Becton Dickinson, Rutherford, New Jersey, USA), allowed to sit for $10 \mathrm{~min}$, then centrifuged at 5000 r.p.m. for $15 \mathrm{~min}$. Plasma total cholesterol was analysed using an Abbott Spectrum Chemistry Analyser (Abbott Laboratories, Abbott Park, Illinois, USA).

\section{Statistical analysis}

All dependent variables were analysed using a repeated measures analysis of variance factorial design. Where significance was found, the least significant difference (LSD) post-hoc test was used. The level of significance was set at $P<0.05$.

\section{Results}

\section{Delayed onset muscle soreness (DOMS) ratings}

No significant treatment effect $(P=0.548)$ or significant treatment by time interaction $(P=0.962)$ was found. A significant time effect was evident $(P=0.0001)$. The LSD post-hoc test revealed that DOMS ratings were significantly elevated $(P<0.0001)$ over baseline levels between 24 (mean(s.e.) 4.15(0.24)) and $96 \mathrm{~h}$ (mean(s.e.) 1.8(0.24)). Both groups showed an increase followed by a steady decrease with peak soreness occurring at $48 \mathrm{~h}$ after exercise (mean(s.e.) 4.7(0.24)). (See Figure 1.)

\section{Creatine kinase (CK)}

The statistical analysis revealed no significant treatment effect between Groups 1 and $2(P=0.295)$, or a significant treatment by time interaction $(P=0.074)$. 
However, there was a significant time effect $(P=$ 0.0001). The LSD post-hoc test revealed that CK was significantly elevated over baseline (mean(s.e.) 95.16(9.90) $\mathrm{Ul}^{-1}$ ) at $48 \mathrm{~h}$ (mean(s.e.) 1410.01(335.13) $\mathrm{Ul}^{-1}$ ) and $72 \mathrm{~h}$ (mean(s.e.) 2361.01(339.71) $\mathrm{Ul}^{-1}$ ) after exercise, at which time CK peaked. CK remained significantly elevated through $144 \mathrm{~h}$ (mean(s.e.) 1063.32(335.13) $\left.\mathrm{Ul}^{-1}\right)$. At $192 \mathrm{~h} \mathrm{CK}$ was still somewhat elevated over baseline (mean(s.e.) 348.22 (335.13) $\mathrm{U}^{-1}$ ), but this difference was not statistically significant. (See Figure 2.)

\section{Strength (1-RM $\left.\mathbf{M}_{\text {conc }}\right)$}

There was no significant treatment effect $(P=0.509)$ or significant interaction effect $(P=0.106)$ but there was a significant time effect $(P=0.0001)$. The greatest reduction in $1-R M_{\text {conc }}$ strength compared with baseline values occurred at $48 \mathrm{~h}$ after exercise in both groups $(P=0.0001)$; this represented a $9 \%$ decrease in strength. Strength remained significantly depressed at $96 \mathrm{~h}$ after exercise by $5.1 \%(P=0.003)$ and at $192 \mathrm{~h}$ after exercise by $3.4 \%(P=0.037)$. (See Figure 3 .)

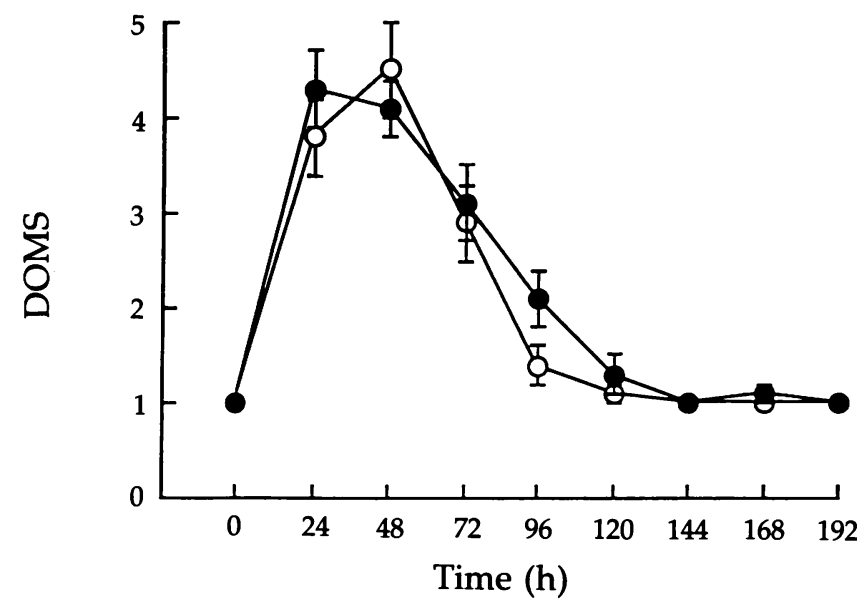

Figure 1. Mean(s.e.) ratings for delayed onset muscle soreness (DOMS) for Group $1(\mathrm{O})$ and Group $2(0)$, across all time periods

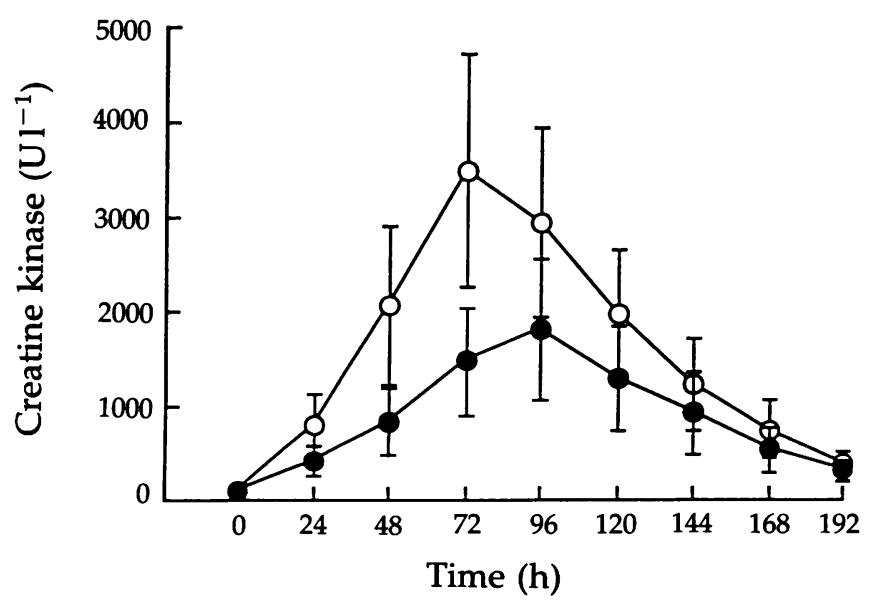

Figure 2. Mean(s.e.) serum creatine kinase (CK) levels for Group $1(O)$ and Group $2(O)$, across all time periods
Repeated eccentrics and muscle soreness: L. L. Smith et al.

\section{Total cholesterol (TC)}

There was no significant group or interaction $(P>0.05)$ effect for TC, suggesting that the two bouts of exercise did not alter the response when compared with one bout of eccentrics. However, there was a significant time effect $(P=0.0001)$. The combined group mean(s.e.) values were $171.500(5.822)$, $161.231(6.015), 158.962(5.485), \quad 156.600(5.922)$ and $160.269(5.232) \mathrm{mg} \mathrm{dl}^{-1}$ before exercise, and at 24, 48, 72 and $96 \mathrm{~h}$ after exercise, respectively. The LSD post-hoc test revealed that TC values were significantly lower than baseline values at $24(P=0.005)$, $48(P=0.0001), 72(P=0.0001)$ and $96 \mathrm{~h}(P=0.0001)$ after exercise. (See Figure 4.)

\section{Discussion}

The present study investigated whether a second bout of eccentric muscle action performed $48 \mathrm{~h}$ after an initial bout would alter the course of delayed muscle soreness (DOMS), serum creatine kinase (CK) and maximum concentric strength (1-RM $\left.\mathrm{R}_{\text {conc }}\right)$. The

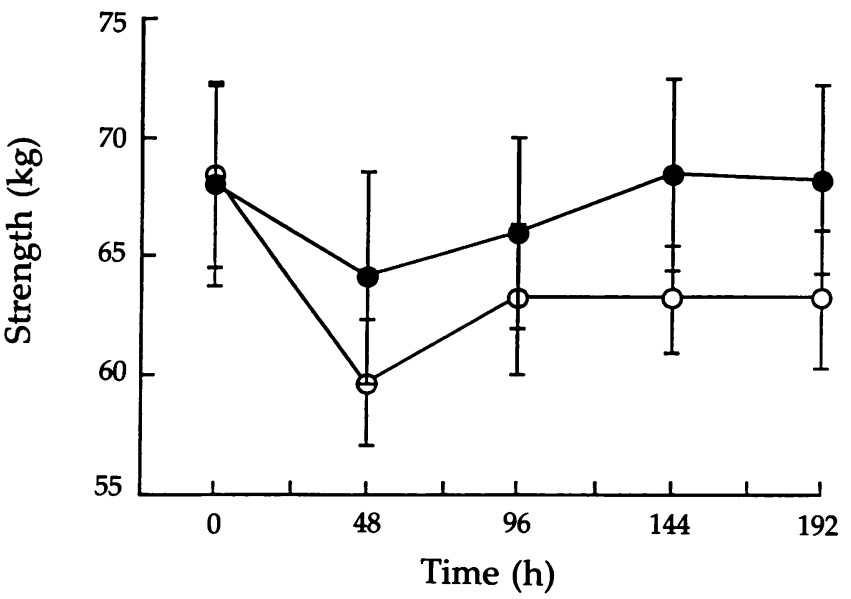

Figure 3. Mean(s.e.) of strength measurements (1-RM $\left.\mathrm{R}_{\text {conc }}\right)$ for Group $1(O)$ and Group $2(O)$, across all time periods

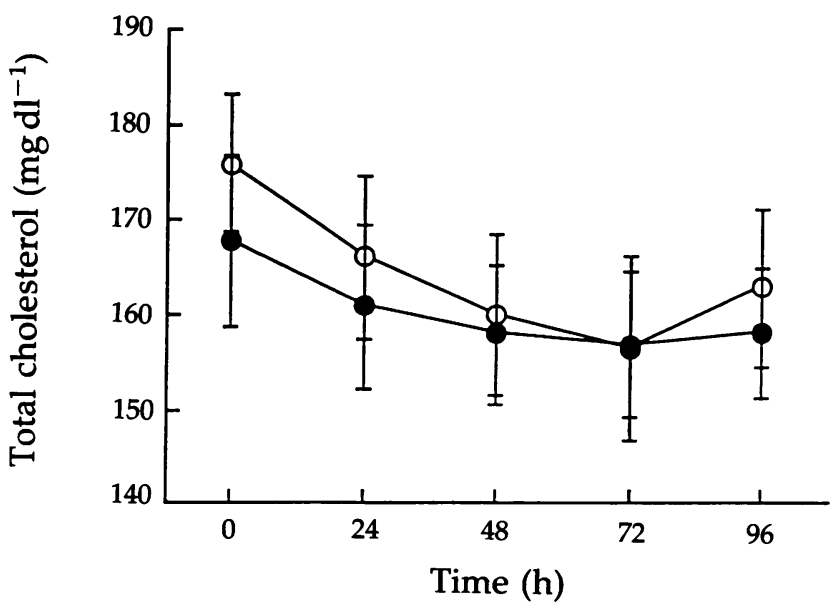

Figure 4. Mean(s.e.) of total cholesterol (TC) for Group 1 (O) and Group 2 (O) 
results revealed no significant differences in the rating of soreness between Group 1 and Group 2, suggesting that an equivalent bout of eccentrics performed $48 \mathrm{~h}$ later does not increase or prolong DOMS (Figure 1). On the other hand, the second bout did not reduce the time course for DOMS, implying that an earlier resolution does not occur in response to this additional bout of eccentric exercise.

The significant time effect for CK seen in this study is similar to what has been reported previously $3,4,16,17$, with $C K$ peaking several days after the eccentric bout of exercise and then gradually returning to baseline. Although there was no significant difference in CK between the two groups, it was interesting to note that Group 1 consistently had higher CK values compared with Group 2. There is considerable individual variability in CK response to eccentric exercise, with some individuals demonstrating an exaggerated response, 'high responders', and others demonstrating a reduced response, 'nonresponders', for the same bout of exercise ${ }^{18}$. Examination of the individual data in the present study did not suggest that Group 1 had a disproportionate number of high $\mathrm{CK}$ responders. A possible explanation for this difference is that Group 1 produced a 'truer maximum' effort during the testing for their 1-RM and consequently performed the exercise at a greater relative percentage of their maximum compared with Group 2. Group 1 also showed a consistently greater loss of strength (although not significantly greater), again suggesting that this group worked harder. Since an increase in $\mathrm{CK}$ after eccentrically biased exercise is taken as indirect evidence of disruption of muscle cell membrane $e^{3,4}$, and since there were no significant group differences, the results of this study suggest that repeating the exercise during the period of soreness produces no additional damage. However, such an interpretation should be made with caution since there is not a good correlation between serum CK and the extent of tissue injury ${ }^{19}$.

The present study revealed no significant treatment effect for strength, suggesting that a repeated bout of eccentrics performed $48 \mathrm{~h}$ after the initial bout, is not deleterious to $1-\mathrm{RM}_{\text {conc. There was, }}$ however, a significant time effect with the largest decrement occurring for both groups at $48 \mathrm{~h}$ after exercise. The literature concurs ${ }^{4,16,20,21}$ that after a bout of unaccustomed eccentrics there is a reduction in strength most likely due to a decline in the inherent capacity of the muscle to produce force ${ }^{1,4}$.

Although a limited amount of research has focused on repeating eccentric exercise $48 \mathrm{~h}$ later, a number of researchers have studied repeated bouts spaced at longer intervals such as 5 and 14 days ${ }^{17}$ and 3,6 and 9 weeks apart ${ }^{22,23}$. All studies concur that changes in DOMS, CK and strength measures are significantly reduced after the second bout of exercise compared with changes after the first bout. Apparently, some 'adaptation' occurs in response to the initial microtrauma and subsequent healing, which than acts to 'protect' the musculature ${ }^{4,16,17}$. It is clear that the adaptation lasts for a considerable amount of time ${ }^{4}$, but it is not known how soon after the initial bout this adaptation occurs. In the present study, if the second bout had resulted in an earlier resolution of DOMS, CK and strength, we could have surmised that an adaptation had occurred; this was not the case. However, the fact that DOMS, CK and strength responses were not exacerbated after Group 2 repeated the exercise, suggests that the 'protective effect' might be present as early as $48 \mathrm{~h}$ after the initial eccentric bout.

An interesting finding of this study was the significant decrease in TC seen for both groups at 24, 48 and $72 \mathrm{~h}$ after exercise (Figure 4). Increased levels of blood cholesterol have been linked with a substantial increase in risk for coronary artery disease (CAD). Although cardiovascular exercise might have some beneficial lowering effects on blood lipids, there is little conclusive evidence about the relationship between muscular strengthening exercise and lipid levels ${ }^{24}$. To the best of our knowledge no strength training studies have investigated acute changes in $\mathrm{TC}$ in response to the eccentric component of weight training. In view of the fact that cholesterol may constitute $13 \%$ of a cell membrane ${ }^{12}$, and that signs of healing have been observed in human subjects as early as $36 \mathrm{~h}$ after eccentric exercise $\mathrm{e}^{25}$, we suggest that the acute decrease in TC in the present study represents the diversion of cholesterol for synthesis of new cell membranes. An alternative or supplementary explanation for the acute reduction in TC could be related to exudative changes which involve the loss of plasma proteins ${ }^{26}$, since swelling, and presumably an increase in exudate, has been reported in association with DOMS ${ }^{9}$.

In conclusion, the results of this study suggest that repeating a bout of exercise during the time of DOMS will not influence the time course of DOMS, serum CK, or strength decrements. Whether or not it might be beneficial or detrimental in terms of other variables, such as factors related to the healing process $^{13,14}$ is presently not known.

\section{References}

1 Newham DJ, Mills KR, Quigley, BM, Edwards RHT. Pain and fatigue after concentric and eccentric muscle contractions. Clin Sci 1983; 64: 55-62.

2 Armstrong RB. Mechanisms of exercise induced delayed onset muscular soreness: a brief review. Med Sci Sports Exerc 1984; 16: 529-38.

3 Armstrong RB. Muscle damage and endurance events. Sports Med 1986; 3: 370-381.

4 Ebbeling CB, Clarkson PM. Exercise induced muscle damage and adaptation. Sports Med 1989; 7: 207-34.

5 Clarkson PM, Fritz VK, Stauber WT. Extracellular matrix disruption in human muscle resulting from eccentric muscle action. (Abstract) Med Sci Sports Exerc 1989; 21(Suppl): S80.

6 Newham DJ, Jones DA, Tolfree SEJ, Edwards RHT. Skeletal muscle damage: a study of isotope uptake, enzyme efflux and pain after stepping. Eur J Appl Physiol 1986; 55: 106-12.

7 Fritz VK, Stauber WT. Characterization of muscles injured by forced lengthening. II. Proteoglycans. Med Sci Sports Exerc 1988; 20: 354-61.

8 Newham DJ, McPhail G, Mills KR, Edwards RHT. Ultrastructural changes after concentric and eccentric contractions of human muscle. J Neur Sci 1983; 61: 109-22.

9 Friden J, Sfakianos PN, Hargens AR, Akeson WH. Residual muscular swelling after repetitive eccentric contractions. J Orthopaedic Res 1988; 6: 493-8.

10 Leadbetter WB. Cell matrix response in tendon injury. Clinics Sports Med 1992; 11: 533-78. 
11 Peacock EE. Wound Repair. 3rd ed. Philadelphia, USA: W. B. Saunders, 1984: 1-14.

12 Guyton AC. Text Book of Medical Physiology. 6th ed. Philadelphia, USA: W. B. Saunders, 1981: 50.

13 Carlson LA, Holmquist L, Lindholm M. Plasma lipid metabolism and trauma: appearance of characteristic apolipoproteins in high density lipoproteins. Acta Chir Scand 1985; 522: 87-106.

14 Man EB, Bettcher PG, Cameron CM, Peters JP. Plasma amino acids, nitrogen and serum lipids of surgical patients. Clin Invest 1946; 25: 701-8.

15 Dons V, Bollerup K, Bonde-Peterson F, Hancke S. The effect of weight-lifting exercise related to muscle fiber composition and muscle cross-sectional area in humans. Eur J Appl Physiol 1979; 40: 95-106.

16 Clarkson PM, Tremblay I. Exercise-induced muscle damage, repair, and adaptation in humans. I Appl Physiol 1988; 65: 1-6.

17 Ebbeling CB, Clarkson PM. Muscle adaptation prior to recovery following eccentric exercise. Eur J Appl Physiol 1990; 60: 26-31.

18 Newham DJ, Jones DA, Edwards RHT. Large delayed plasma creatine kinase changes after stepping exercise. Muscle Nerve 1983; 6: 380-5.
Repeated eccentrics and muscle soreness: $L$. L. Smith et al.

19 Van Der Meulen JH, Kuipers H, Drukker J. Relationship between exercise-induced muscle damage and enzyme release in rats. J Appl Physiol 1991; 71: 999-1004.

20 Francis K, Hoobler T. Delayed onset muscle soreness and decreased isokinetic strength. J Appl Sports Sci Res 1988; 2: 20-3.

21 Talag TS. Residual muscular soreness as influenced by concentric, eccentric and static contractions. Research Quarterly 1972; 44: 458-69.

22 Byrnes WC, Clarkson PM, White JS et al. Delayed onset muscle soreness following repeated bouts of downhill running. I Appl Physiol 1985; 59: 710-15.

23 Triffletti P, Litchfield PE, Clarkson PM, Byrnes WC. Creatine kinase and muscle soreness after repeated isometric exercise. Med Sci Sports Exerc 1988; 20: 242-8.

24 Kokkinos PF, Hurley BF. Strength training and lipoprotein-lipid profiles. Sports Med 1990; 9: 266-72.

25 Friden J, Sjostrom M, Ekblom B. Myofibrillar damage following intense eccentric exercise in man. Int J Sports Med 1983; 4: 170-6.

26 Keele KD, Stern PRS. Serum lipid changes in relation to pain. J R Coll Physicians Lond 1973; 7: 319-29.

\title{
Announcement
}

\section{Relocation of Journal Publishing Office}

\author{
As from 1st October 1994, the publishing, editorial, production and reprint offices \\ are moving to: \\ Elsevier Science Ltd \\ The Boulevard, Langford Lane, \\ Kidlington, Oxford, OX5 1GB, UK \\ Main switchboard: \\ Tel: $+44(0) 1865843000$ \\ Fax: $+44(0) 1865843010$
}

All correspondence and queries relating to the journal should be sent to this address

Newly submitted manuscripts should be mailed to the Editor 